\title{
Membrane Biochips
}

\author{
Ye Fang, Anthony G. Frutos, Brian Webb, Yulong Hong, Ann Ferrie, Fang Lai, and Joydeep Lahiri \\ Biochemical Sciences, Science and Technology Division, Corning Incorporated, Corning, NY, USA
}

\section{ABSTRACT}

Membrane-bound proteins represent the single most important class of drug targets. This article discusses the issues surrounding fabrication of membrane-protein microarrays by conventional robotic pin printing techniques. Ligand binding selectivity and specificity to $G$ protein-coupled receptor (GPCR) microarrays are presented. The potential applications of these arrays for drug screening are discussed.

\section{INTRODUCTION}

Gene expression analysis using DNA microarrays has demonstrated the value of a global approach to understanding biological systems (6). The correspondence between mRNA levels and protein abundance is complicated by mRNA splicing and posttranslational modifications. Presumably, protein microarrays $(18,19)$ (e.g., antibodies to the yeast proteome) will offer a more accurate estimation of protein abundance. In addition to profiling protein abundance, however, protein arrays can be used for the multiplexed investigation of protein-protein and protein-small molecule interactions. Since proteins are typically what drugs are designed against, protein arrays are uniquely suited for obtaining information about compound design and selectivity (17). Nevertheless, the use of protein microarrays to obtain "universal" information about compound affinity and selectivity up front in the drug discovery process has not been realized. In part, there are significant technical hurdles that need to be overcome before protein microarrays become a standard tool in drug discovery. More significantly, there is a conflict between conventional drug discovery strategies and the use of protein arrays for compound screening. Traditionally, following the identification of a particular protein "target," libraries of compounds are tested for their ability to bind to that particular target. Selectivity studies are carried out further downstream, after the identification of a "hit" compound and in its progression to a "lead." In principle, protein microarrays can be used to bridge primary and secondary screening efforts by offering selectivity information naturally and with amounts of sample and reagents that are typically lower than that required for conventional microplate assays.

Nowhere is the ability to screen compounds against multiple proteins more pertinent than for the development of drugs against $\mathrm{G}$ protein-coupled receptors (GPCRs) and other membrane-bound targets. Approximately $50 \%$ of current drug targets are membrane bound; $20 \%$ of the top 200 best selling drugs target GPCRs (5). Structurally, GPCRs contain seven transmembrane helices, a glycosylated extracellular $\mathrm{N}$ terminus and an intracellular C terminus. GPCRs mediate key cellular processes through the binding of ligands to the extracellular side of the receptor, which leads to activation of $\mathrm{G}$ proteins associated with the receptor on the cytosolic side (11). There are an estimated 400-700 GPCRs, approximately 190 of which have known ligands (13). GPCRs for which ligands are unidentified are termed orphan GPCRs, many of which are presumed to be important drug targets. Given the large number of GPCRs, many of which bind identical ligands and yet carry out different tissue- or stimuli-specific functions, there is a compelling need to develop technologies that naturally offer multiplexing. There are many technologies available for screening compounds against GPCRs (11); none, however, offer the ability to simultaneously screen a compound against multiple GPCRs in a single assay.

\section{MEMBRANE MICROARRAYS: KEY ISSUES}

Fabricating microarrays of proteins associated with membranes is challenging $(3,17)$. There are three significant issues that are unique to membrane protein microarrays. First, unlike DNA or conventional protein arrays, fabricating microarrays containing GPCRs requires surface chemistries that enable spatial localization of both the GPCR and the associated lipid molecules to within the printed microspots. Second, covalent immobilization of the entire membrane is not desirable, because lateral mobility is an intrinsic and physiologically important property of a biological membrane (10). While the importance of preserving long-range fluidity in a supported membrane microspot for compound screening assays is unclear, the ability to preserve long-range fluidity without compromising assay performance can be presumed to be advantageous. Third, the membrane needs to be offset from the surface to accommodate the extramembrane domains of the bound proteins. Surfaces presenting amphiphilic tethers or polymer "cushions" (8) offer the possibility of incorporating membrane proteins in their correctly folded conformations.

We recently described the fabrication of microarrays of GPCRs on surfaces coated with $\gamma$-aminopropylsilane (GAPS) and described their use for ligand screening assays (9). We were interested in surfaces on which membranes exhibited high me- 
chanical stability, while preserving long-range lateral fluidity. These properties are contradictory. Strong membrane-substrate interactions, such as those afforded through covalent immobilization, would preclude lateral diffusion of molecules in the microspot, and weak interactions with the substrate would lead to poor mechanical stability. Mechanical stability is a serious concern, as supported membranes are vulnerable to physical degradation; supported lipids on bare glass spontaneously desorb when withdrawn through air-water interfaces (4). Assays using microarrays involve incubations with different buffers and sample solutions, which involve repeated withdrawals through

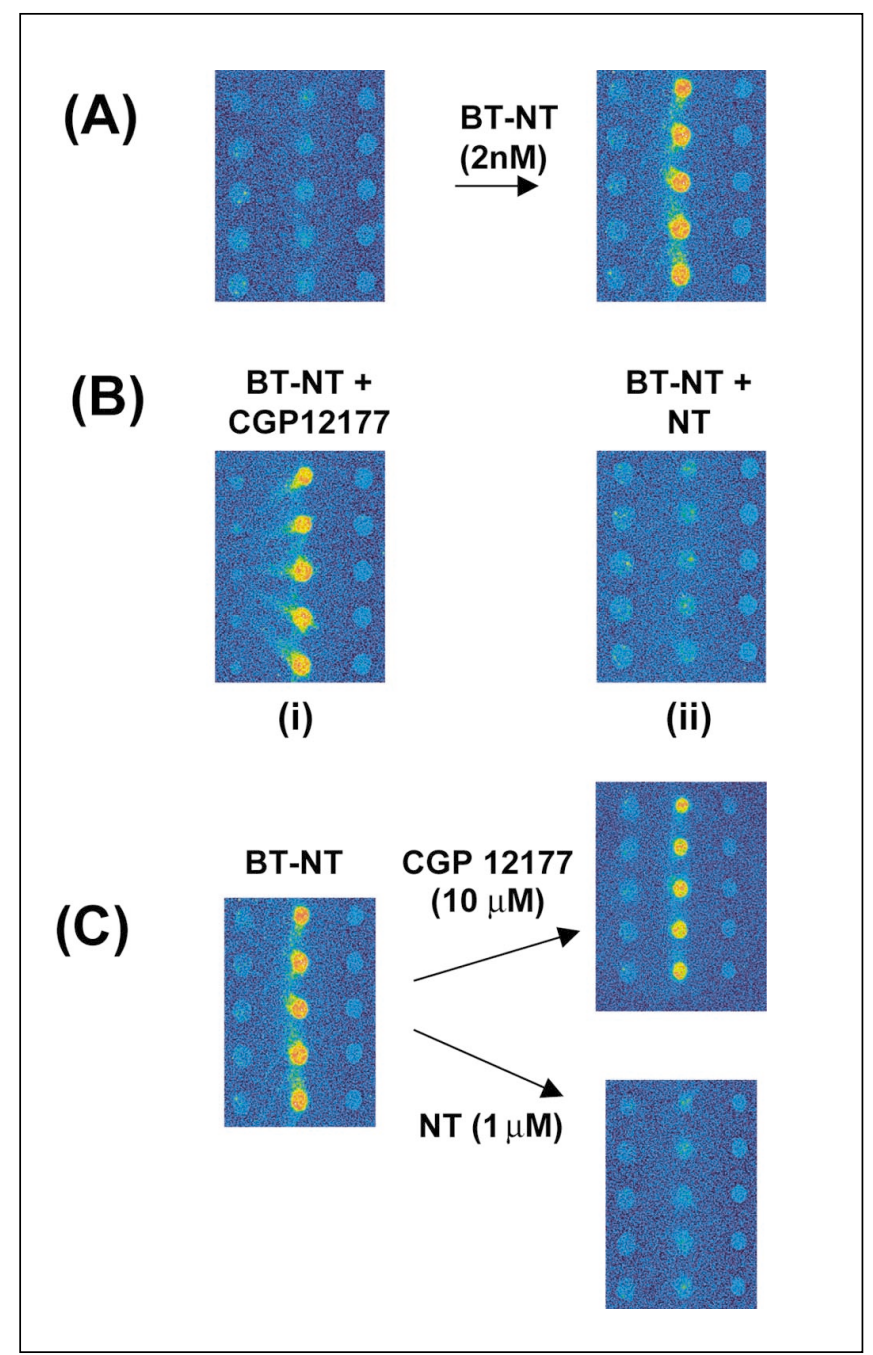

Figure 1. Competitive binding and displacement of ligands to GPCR microarrays. Multiple arrays of the $\beta 1$ ( $1^{\text {st }}$ column), NTR1 ( $2^{\text {nd }}$ column), and D1( $3^{\text {rd }}$ column) receptors were fabricated by robotic pin printing on a GAPS slide. Each array contains five replicate microspots of each receptor. (A) Fluorescence images of the microarray before and after incubation with BT-NT $(2 \mathrm{nM})$. (B) Fluorescence images showing the competitive binding of BT-NT $(2 \mathrm{nM})$ and unlabeled compounds to the microarray. The unlabeled ligands were (i) CGP12177 (1 $\mu \mathrm{M})$ or (ii) NT $(1 \mu \mathrm{M})$. (C) Fluorescence images showing the displacement of prebound BT-NT by unlabeled compounds. The microarray was first incubated with a solution of BT-NT $(2 \mathrm{nM})$, washed, dried, and then imaged. This was followed by incubation with a second solution containing either CGP12177 $(10 \mu \mathrm{M})$ or NT $(1 \mu \mathrm{M})$. For all assays, the binding buffer consisted of $50 \mathrm{mM}$ Tris- $\mathrm{HCl}(\mathrm{pH}$ 7.4), $10 \mathrm{mM} \mathrm{MgCl}_{2}, 2 \mathrm{mM}$ EDTA, and $0.1 \%$ bovine serum albumin (BSA). buffer-air interfaces during these multiple steps. It is, therefore, important to choose substrates on which supported membranes resist physical removal upon exposure to air. GAPS-coated surfaces provide an optimal balance; supported membranes exhibit long-range lateral fluidity, albeit reduced relative to bare glass substrates, and are also resistant to desorption, independent of the phase (gel or fluid phase) of the lipid (9). The nature of the GAPS surface and other amine containing surfaces, e.g., polyethyleneimine (15), is itself important for optimal assay performance; the methods of deposition, the extent to which the siloxyl groups are cross-linked, the amine surface density, and the overall surface hydrophobicity are some of the factors or metrics that determine performance.

\section{ARRAY FABRICATION}

The fabrication of microarrays was carried out using a quill pin printer (Cartesian Technologies, Irvine, CA, USA) (9). We have not investigated other printing techniques, such as those involving solid pins, ink-jet printers, etc. We believe that other printing techniques should be feasible. There may, however, be issues with particular printing methods; for example, thermal ink-jet printing may denature proteins in the membrane or lead to phase transitions of the membrane itself.

In a typical print run, $10 \mu \mathrm{L}$ of probe membrane solutions were added to different wells of a 384-well microplate. A single insertion of the pin into the solution can yield up to 400 identical spots, with coefficient of variation $(\mathrm{CV})$, due to printing, $<7 \%$ across the spots. This amount of membrane solution corresponds to about $0.5 \mathrm{~nL} /$ microspot and effectively demonstrates the assay miniaturization afforded by microarray technology. After printing, the arrays were incubated in a humid chamber at room temperature for 1 hour and then used for ligand binding experiments. For longer-term storage, the arrays were stored at $4^{\circ} \mathrm{C}$ in a dessicator filled with nitrogen. Ligand binding experiments to test the shelf-life of the microarrays demonstrated negligible loss in activity over the 2-month duration of the study.

\section{COMPOUND SCREENING}

The screening of compounds using GPCR microarrays can be carried out in many different formats and with different types of GPCR probes. For example, compounds could be tested against an array consisting of one member of each GPCR family or against an array consisting of all of the GPCRs within a family (e.g., the adrenergic receptors) or against a full index GPCR array. Ligand fishing for orphan GPCRs could also be carried out using GPCR microarrays. Arrays could be printed on a slide, the bottoms of microplates, or even on a chip with an integrated fluidic interface. The assays described below were carried out on slides, on which GPCR arrays were printed in replicate (typically 16 arrays on a $1-\times 3$-inch slide), with each array being treated with different compounds or compounds at different concentrations. We used fluorescence detection because of the availability of high-resolution fluorescence scanners developed for DNA microarrays. A limitation to using fluorescence detection is the limited availability of labeled ligands for competition or ligand fish- 
ing assays. We believe that radioactivity-based detection, using readily available radiolabeled ligands, should be feasible, especially with the advent of high-resolution phosphor imagers (e.g., Typhoon $^{\mathrm{TM}}$ 9410; Amersham Biosciences, Piscataway, NJ, USA) that provides adequate resolution for imaging microarrays.

\section{Screening Across Different Families}

To demonstrate the feasibility of using GPCR microarrays to test compounds across different GPCR families, we printed microarrays consisting of the adrenergic receptor $(\beta 1)$, the neurotensin receptor (NTR1), and the dopamine receptor (D1). To carry out competitive binding assays, we first had to demonstrate the specific binding of labeled ligands to the array. As a test case, we used fluorescently labeled neurotensin (NT), (Bodipy ${ }^{\circledR}$-TMR-NT [BT-NT]; PerkinElmer, Boston, MA, USA), the Bodipy-TMR-labeled analog of NT, and the cognate ligand for NTR1. Figure 1A shows fluorescence images of microarrays treated with BT-NT $(2 \mathrm{nM})$, and specific binding to the column of microspots corresponding to NTR1 is observed.

Using NTR1 microarrays, we verified that the affinity of BTNT for NTR1 was approximately $1.2 \mathrm{nM}$, in accordance with the literature (1). This tight binding affords the possibility of carrying out the competition assay in two distinct formats. The first method involves incubation of the array with solutions containing BT-NT and unlabeled test compounds. Figure 1B shows images of the array treated with solutions containing BT-NT (2 $\mathrm{nM})$ and either CGP12177 $(1 \mu \mathrm{M})$ or unlabeled NT $(1 \mu \mathrm{M})$. CGP12177 is known to not bind to NTR1 and no inhibition is expected (Figure 1B [i]). NT binds to NTR1 with high affinity $\left(\mathrm{K}_{\mathrm{d}}\right.$ approximately $\left.1 \mathrm{nM}\right)$, and complete inhibition is expected, in accordance with our data (Figure 1B [ii]). The second type of competition assay involves preincubation of the microarray with the labeled ligand prior to its being treated with solutions containing putative ligands. This assay format is feasible because of the high affinity of BT-NT to NTR1; mass transport limited rebinding (14) greatly reduces the rate of dissociation of BT-NT, such that the binding is essentially irreversible over the duration of the assay. Displacement of the bound ligand can, however, be readily effected by treatment of the array with solutions contain-

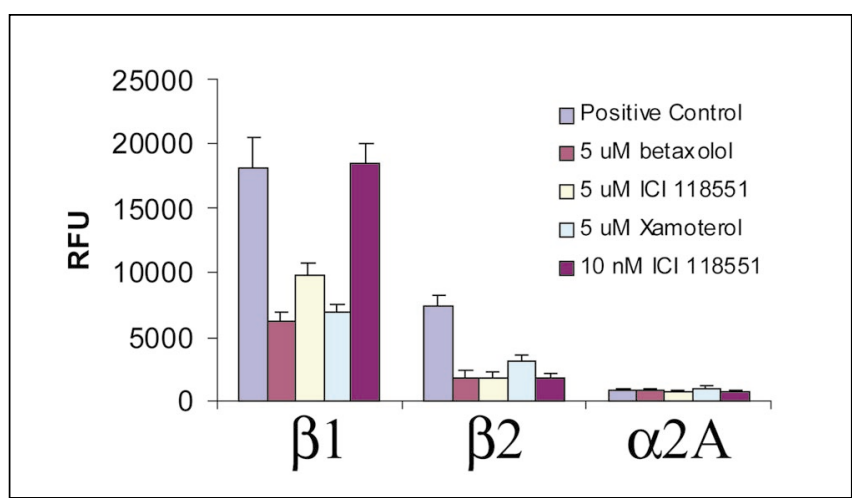

Figure 2. Histogram analysis of BT-CGP binding to arrays of the adrenergic receptor. Five separate arrays of the $\beta 1, \beta 2$, and $\alpha 2 \mathrm{~A}$ receptors were printed on a single GAPS-coated gold slide. The arrays were incubated with a solution containing $2 \mathrm{nM}$ BT-CGP in the absence (positive control) and presence of betaxolol $(5 \mu \mathrm{M})$, ICI $11851(5 \mu \mathrm{M})$, xamaterol $(5 \mu \mathrm{M})$, and ICI $118551(10 \mathrm{nM})$. ing a competitive ligand. Figure $1 \mathrm{C}$ (top) shows an image of an array with bound BT-NT treated with a solution containing CGP12177; the small decrease in the fluorescence signal is similar to that obtained when the array is treated with buffer only. Figure 1C (bottom) shows an image of an identical array being treated with a solution containing NT. The image shows that the bound BT-NT is completely displaced, in agreement with the literature and the previously described assay. The data also demonstrates that the binding of BT-NT is fully reversible. These experiments demonstrate the feasibility of using GPCR array technology to test compounds across different GPCR families.

\section{Screening Within One Family}

GPCR microarrays can be used for estimating the relative affinity of a compound between different members of a given family, i.e., an assay that is relevant for the design of selective drugs. To test this application, we printed arrays of the adrenergic receptor. Each array contained the $\beta 1$, the $\beta 2$, and the $\alpha 2 \mathrm{~A}$ subtypes. Competition experiments were carried out using by incubating the array with a solution containing fluorescently labeled CGP12177 (Bodipy-TMR-CGP12177 [BT-CGP12177]), a $\beta$-selective ligand, and various unlabeled compounds (betaxolol, xamoterol, and ICI 118551). The inhibitors were used at micromolar concentrations. BT-CGP12177 was present at a concentration of $2 \mathrm{nM}\left(\mathrm{K}_{\mathrm{d}}\right.$ approximately $1 \mathrm{nM}$ for $\beta 1$ and $\beta 2)$ (12). At micromolar concentrations of potential ligands, one expects to merely screen for inhibition; ideally, demonstrating subfamily selectivity may require testing inhibitors (or structurally modified analogs) at lower concentrations. We, therefore, also used ICI 118551 at a concentration of $10 \mathrm{nM}$. This compound binds the $\beta 1$ and $\beta 2$ receptors with $K_{i}$ values of approximately $120 \mathrm{nM}$ and $1.2 \mathrm{nM}$, respectively (2). Figure 2 shows the results of these experiments. The histograms represent the background subtracted fluorescent signals (relative fluorescence units [RFU]) for the $\beta 1, \beta 2$, and $\alpha 2 \mathrm{~A}$ microspots upon treatment

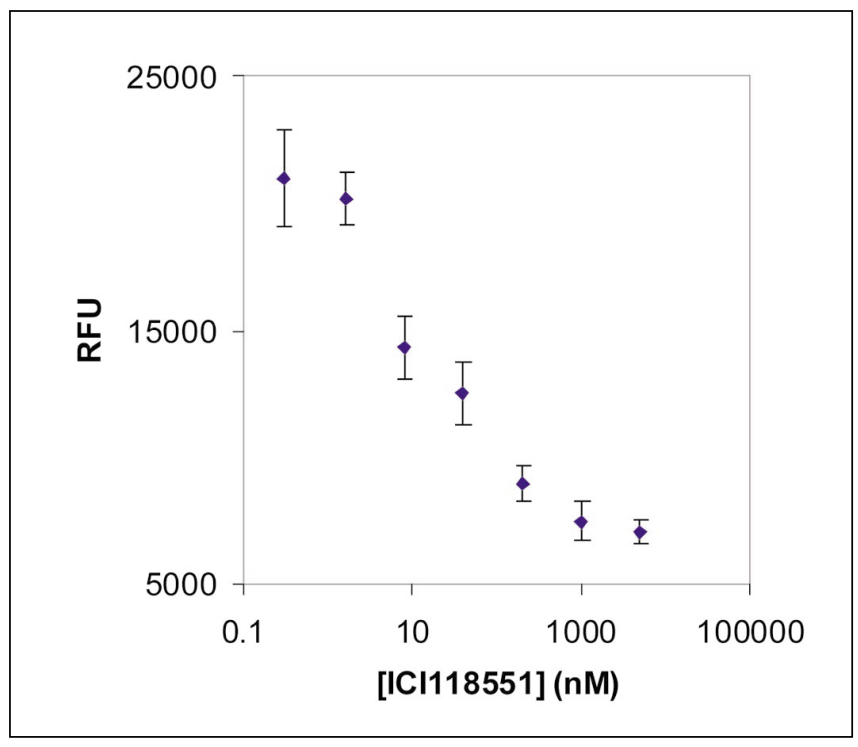

Figure 3. Concentration-dependent inhibition of binding of BT-CGP12177 by ICI 118551 to microarrays of the $\beta 1$ receptor. 
with the various compounds. The expected $\beta$-selectivity of BTCGP12177 is clearly observed. Betaxolol, ICI 118551, and xamoterol, are all known inhibitors $(12,16)$. The observed inhibition demonstrates the ability to screen potential inhibitors within a GPCR family. The differential affinities of these inhibitors for the GPCRs are swamped out at micromolar concentrations, in agreement with the known high affinities of these compounds. The differential affinity of ICI118551 for the $\beta 1$ and $\beta 2$ receptors is observed at $10 \mathrm{nM}$; while negligible inhibition is observed for the $\beta 1$ receptor, approximately $74 \%$ inhibition is observed for the $\beta 2$ receptor. These experiments clearly demonstrate that GPCR microarrays are a powerful tool to test compound selectivity across closely related GPCRs.

\section{Estimating Compound Affinities}

While microarrays are well suited for screening across multiple proteins, estimating compound affinities using microarrays is tricky. These estimations may require measuring small $(<2-$ fold) differences in signal intensity, which requires significantly greater precision during printing, the assay itself, and data analysis. The ability to obtain binding constants using microarrays almost serves as a litmus test for the robustness of a microarraybased assay. There is a second significant consideration that is particular to GPCR microarrays. The affinity of a compound for a GPCR depends on whether the GPCR is complexed to a $\mathrm{G}$ protein (11). If the affinity of a compound using a microarray-based assay is the same as that observed using a solutionbased assay, we can assume that the relative proportions of complexed and uncomplexed GPCRs does not change as a result of immobilization on the surface.

We fabricated microarrays, in replicate, of the $\beta 1$ adrenergic receptor. Each array was treated a solution containing a fixed concentration of BT-CGP12177 (1 nM) and different concentrations of ICI 118551. From a plot of the inhibition profile compounds (see Figure 3), we estimate that the $\mathrm{K}_{\mathrm{i}}$ value for ICI 118551 is $25 \mathrm{nM}$, which is in reasonable agreement with the literature (2). We have previously carried similar experiments using the NTR1 (9) and have estimated binding constants that were in accordance with literature reports. While it is possible that there are GPCRs, which, upon immobilization, would result in artifacts (e.g., structural changes) leading to incorrect estimations of ligand affinity, these experiments certainly demonstrate the ability to obtain compound affinities. Coupled with the ability to test multiple GPCRs in parallel, these experiments also suggest the viability of using GPCR microarrays for highly multiplexed studies of compound potency.

\section{CONCLUSION}

We have demonstrated the fabrication of GPCR microarrays and their use for compound screening. The technology is powerful. It offers multiplexing, minimal sample usage, archivability of precious GPCRs (printed GPCRs are stable for months), and is amenable to high-throughput screening platforms, such as microplates. The technology can be extended to other membrane bound targets; we have already demonstrated the fabrication of arrays of gangliosides (7) and the epidermal growth fac- tor (EGF) receptor. The next biggest challenge for GPCR microarrays is whether their use can be extended beyond compound screening. Can they be used to classify compounds as agonists or antagonists? Experiments for estimating compound affinities suggest that the GPCR/G protein complex is preserved in the microspot, suggesting that these assays may be feasible. If successful, the assays may result in a step change in GPCR screening technology and demonstrate that protein arrays in general may be used to screen for both affinity and function.

\section{REFERENCES}

1.Barroso, S., R. Francoise, D. Nicolas-Etheve, J.-L. Reversat, J.-M. Bernassau, P. Kitabgi, and C. Labbe-Jullie. 2000. Identification of residues involved in neurotensin binding and modeling of the agonist binding site in neurotensin receptor 1. J. Biol. Chem. 275:328-336.

2.Bilski, A., S. Dorries, J.D. Fitzgerald, R. Jessup, H. Tucker, and J. Wale. 1980. ICI 118551, a potent b2 adrenoreceptor antagonist. Br. J. Pharmacol. 69:292

3.Bowie, J.U. 2001. Stabilizing membrane proteins. Curr. Opin. Struct. Biol. 11:397-402.

4.Cremer, P.S. and S.G. Boxer. 1999. Formation and spreading of lipid bilayers on planar glass supports. J. Phys. Chem. B 103:2554-2559.

5.Drews, J. 2000. Drug discovery: a historical perspective. Science 287:19601963.

6.Eisen, M.B. and P.O. Brown. 1999. DNA arrays for analysis of gene expression. Methods Enzymol. 303:179-205.

7.Fang, Y., A.G. Frutos, and J. Lahiri. Ganglioside microarrays for toxin detection. (In press.)

8.Fang, Y., A.G. Frutos, and J. Lahiri. 2002. G protein-coupled receptor microarrays. Chembiochem. 3:987-991.

9.Fang, Y., A.G. Frutos, and J. Lahiri. 2002. Membrane protein microarrays. J. Am. Chem. Soc. 124:2394-2395.

10.Groves, J.T. and S.G. Boxer. 2002. Micropattern formation in supported lipid membranes. Acc. Chem. Res. 35:149-157.

11.Haga, T. and G. Berstein (Eds.). 1999. G Protein-Coupled Receptors. CRC Press, Boca Raton.

12.Heithier, H., D. Hallmann, F. Boege, H. Reilaender, C. Dees, K.A. Jaeggi, D. Arndt-Jovin, T.M. Jovin, and E.J.M. Helmreich. 1994. Synthesis and properties of fluorescent beta-adrenoceptor ligands. Biochemistry 33:91269134.

13.Howard, A.D., G. McAllister, S.D. Feighner, Q. Liu, R.P. Nargund, L.H.T Van der Ploeg, and A.A. Patchett. 2001. Orphan G-protein coupled receptors and natural ligand discovery. Trends Pharmacol. Sci. 22:132-140.

14.Lahiri, J., L. Isaacs, B. Grzybowski, J.D. Carbeck, and G.M. Whitesides. 1999. Biospecific binding of carbonic anhydrase to mixed SAMS presenting benzenesulfonamide ligands: a model system for studying lateral steric effects. Langmuir 15:7186-7198.

15.Majewski, J., J.Y. Wong, C.K. Park, M. Seitz, J. Israelachvili, and G.S. Smith. 1998. Structural studies of polymer-cushioned lipid bilayers. Biophys. J. 75:2363-2367.

16.Malta, E., M. Mian, and C. Raper. 1985. The in vitro pharmacology of xamoterol (ICI 118,587). Br. J. Pharmacol. 85:179-187.

17.Mitchell, P. 2002. A perspective on protein microarrays. Nat. Biotechnol. 20:225-229.

18.Zhu, H., M. Bilgin, R. Bangham, D. Hall, A. Casamayor, P. Bertone, N. Lan, R. Jansen, et al. 2001. Global analysis of protein activities using protein chips. Science 293:2101-2105.

19.Zhu, H. and M. Snyder. 2001. Protein arrays and microarrays. Curr. Opin. Chem. Biol. 5:40-45.

\section{Address correspondence to:}

Dr. Joydeep Lahiri

Biochemical Sciences

Science and Technology Division

Corning Incorporated

Corning, NY, 14831 USA

e-mail:lahirij@corning.com 